\title{
NON-LINEAR GENERALIZED MAXWELL MODEL FOR DYNAMIC CHARACTERIZATION OF VISCOELASTIC COMPONENTS AND PARAMETRIC IDENTIFICATION TECHNIQUES
}

\author{
HANEN JRAD* \\ Laboratoire d'Ingénierie des Systèmes \\ Mécaniques et des Matériaux (LISMMA), Institut \\ Supérieur de Mécanique de Paris, 3 rue Fernand \\ Hainaut, 93407 Saint Ouen Cedex, Paris, France \\ Unité Modélisation, Mécanique et Productique \\ (U2MP), Ecole Nationale d'Ingénieurs de Sfax, BP \\ $N^{\circ} 1173$, Sfax, 3038, Tunisia \\ Email : hanen.jrad@supmeca.fr
}

\author{
JEAN LUC DION \\ Laboratoire d'Ingénierie des Systèmes \\ Mécaniques et des Matériaux (LISMMA), Institut \\ Supérieur de Mécanique de Paris, 3 rue Fernand \\ Hainaut, 93407 Saint Ouen Cedex, Paris, France \\ Email : jean-luc.dion@supmeca.fr
}

\author{
FRANCK RENAUD \\ Laboratoire d'Ingénierie des \\ Systèmes Mécaniques et des \\ Matériaux (LISMMA), Institut \\ Supérieur de Mécanique de Paris, 3 \\ rue Fernand Hainaut, 93407 Saint \\ Ouen Cedex, Paris, France \\ Email : franck.renaud@supmeca.fr
}

\author{
IMAD TAWFIQ \\ Laboratoire d'Ingénierie des \\ Systèmes Mécaniques et des \\ Matériaux (LISMMA), Institut \\ Supérieur de Mécanique de Paris, 3 \\ rue Fernand Hainaut, 93407 Saint \\ Ouen Cedex, Paris, France \\ Email : imad.tawfiq@supmeca.fr
}

\author{
MOHAMED HADDAR \\ Unité Modélisation, Mécanique et \\ Productique (U2MP), Ecole \\ Nationale d'Ingénieurs de Sfax, BP \\ N¹173, Sfax, 3038, Tunisia \\ Email : \\ mohamed.haddar@enis.rnu.tn
}

\begin{abstract}
Viscoelastic components are incorporated into automobile and aerospace structures system in order to damp mechanical vibrations. Viscoelastic components are a key element in designing desired dynamic behaviour of mechanical systems. Viscoelastic components dynamic characteristics are often very complex, due to the dependence of its response on several variables, such as frequency, amplitude, preload, and temperature. These dependencies can be critical in capturing the mechanical proprieties and so non linear dynamical behaviour may appear. Assuming that non linearities are due to non linear elasticity, the non linear Generalized Maxwell Model (GMM) is proposed to characterize dynamics of viscoelastic components. Parameters of GMM are identified from Dynamic Mechanical Analysis (DMA) tests for different excitation frequencies. A particular result from identification is that the non linear stiffness is dependent upon displacement amplitude and static displacement under static preload. The
\end{abstract}

significance of this result is that the non linear dynamics of the viscoelastic component can be represented by a simple analytical model capable to produce accurate results. Comparison between measurements and simulations of dynamic stiffness of viscoelastic component has been carried on.

Keywords: Viscoelastic components, Dynamic behaviour, Nonlinear GMM, Parametric identification.

\section{NOMENCLATURE}

$x$

$x_{00}$

$x_{0}$

$x_{d}$

$x_{0, h}$

$x_{d, h}$ displacement and $\hat{x}$ its Fourier transform displacement amplitude

displacement under static preload amplitude of sinusoidal displacement displacement under static preload for $h^{\text {th }}$ test, $h=[1 . . \mathrm{Ne}]$

amplitude of sinusoidal displacement for $h^{\text {th }}$ test, $h=[1 . . \mathrm{Ne}]$

*hanen.jrad@supmeca.fr 


\begin{tabular}{|c|c|}
\hline$F$ & force and $\hat{F}$ its Fourier transform \\
\hline$F_{00}$ & force amplitude \\
\hline$F_{0}$ & resorting force of the non-linear spring \\
\hline$F_{i}$ & $\begin{array}{l}\text { resorting force of the } i^{\text {th }} \text { Maxwell cell, } \\
i=[1 . . N]\end{array}$ \\
\hline$H_{0}$ & height of the cylindrical rubber sample \\
\hline$D_{0}=2 R_{0}$ & Diameter of the cylindrical rubber sample \\
\hline$S_{0}$ & Section of the upper cylindrical rubber \\
\hline$\Delta H$ & displacement \\
\hline$N$ & Number of Maxwell cells \\
\hline$P$ & static preloads \\
\hline$A$ & amplitude of sinusoidal displacements \\
\hline$K^{*}$ & dynamic stiffness \\
\hline$K^{\prime}$ & real part part of dynamic stiffness \\
\hline$K^{\prime \prime}$ & imaginary part of dynamic stiffness \\
\hline$K_{m}^{*}$ & measured dynamic stiffness \\
\hline$K_{0}$ & stiffness of non linear spring \\
\hline$K_{m 0}$ & measured stiffness of non linear spring \\
\hline $\bar{K}_{0}$ & $\begin{array}{l}\text { mean value of estimated stiffness of non } \\
\text { linear spring }\end{array}$ \\
\hline$K_{0, h}$ & measured stiffness for $h^{\text {th }}$ test, $h=[1 . . \mathrm{Ne}]$ \\
\hline$K_{i}$ & stiffness of the $i^{\text {th }}$ spring \\
\hline$C_{i}$ & damping of the $i^{\text {th }}$ dashpot \\
\hline$\omega$ & angular frequency \\
\hline$\omega_{z, i}$ & zero of the $i^{\text {th }}$ Pole-Zero couple \\
\hline$\omega_{p, i}$ & pole of the $i^{\text {th }}$ Pole-Zero couple \\
\hline$\varphi$ & dephasing phase \\
\hline$\phi$ & mean phase angle for all tests \\
\hline$f_{1}$ & upper bound of the frequency domain \\
\hline$f_{2}$ & lower bound of the frequency domain \\
\hline$\alpha, \beta, \gamma, \lambda$ and $\theta$ & constants \\
\hline$\delta_{i j}$ & kronecker's symbol \\
\hline$N$ & number of Maxwell cells \\
\hline $\mathrm{Ne}$ & number of tests \\
\hline$Z(\omega)=|Z(\omega)| e^{i \varphi(\omega)}$ & dynamic stiffness as a function of frequency \\
\hline$|Z(\omega)|$ & $\begin{array}{l}\text { dynamic stiffness magnitude as a function of } \\
\text { frequency }\end{array}$ \\
\hline$\varphi(\omega)$ & $\begin{array}{l}\text { dynamic stiffness angle phase as a function } \\
\text { of frequency }\end{array}$ \\
\hline
\end{tabular}

\section{INTRODUCTION}

Viscoelastic components, which have substantial energy absorption abilities, are always incorporated into automobile and aerospace structure systems in order to damp mechanical vibrations and thus avoid serious damage. Viscoelasticity is widely studied since decades: considering works of $[1,3,7,10,15,28]$. Viscoelasticity is a causal phenomenon for which the force always precedes the displacement. This behaviour can be described by the relaxation function or the creep function. In the Fourier domain, the dynamic stiffness is a complex function which depends on frequency.

Several experimental studies have been carried out to characterize viscoelastic behavior providing important results and understanding of viscoelastic components dynamics. Oberst and Frankenfeld [20] proposed to study the first mode of a sandwich beam consisting of metal skins and a viscoelastic core. Their method allows knowing the damping induced by the viscoelastic core at the frequency of the mode. Several authors like Barbosa and Farage [2] and Castello et al. [4] used this kind of technique for viscoelastic parameters identification. It is also possible to deduce the mechanical properties of a viscoelastic component from different measures of natural frequencies of a simple form sample like a beam for example, see Chevalier [6]. These methods use Frequency Response Function (FRF), hence, they can only characterize the frequencies of modes and not on a wide frequency band. Moreover, these methods are valid under the assumption of linear material excitation amplitude. Chen [5] suggested measuring directly the relaxation functions and creep to deduce the coefficients of a series of Prony. However, this is very efficient to get values at low frequency, when the material takes time to respond to the excitation. But to get high frequency values, a perfect unit step function is required to assess when exciting the material, which is technically hard. The most suitable kind of test is the Dynamic Mechanical Analysis (DMA), it is a useful technique for acquiring knowledge on the behaviour of a material versus frequency. A DMA tester is used in this work to determine the dynamic stiffness of the viscoelastic component depending on the frequency.

Moreover, viscoelastic components are a key element in designing desired dynamic behaviour of mechanical systems; therefore, different models describing viscoelastic behaviour have been developed. Gaul et al. [12] presented the constant complex modulus model which is non-causal model, it is only suitable in the frequency domain, but, it is not a relevant model since its modulus is constant. Maxwell model represented by Park [22] as a spring and dash-pot connected in series and Kevin Voigt model which consists of a spring and dash-pot in parallel, are efficient only on a small frequency range. In fact, they are unrealistic respectively at low and high frequencies, where their modulus is respectively: infinitely small and high and the dynamic stiffness phase angle of the Kevin Voigt model is linearly dependent of frequency. The Zener model, see Huynh et al.[13], underestimates the dynamic stiffness at low frequencies and overestimates it at high frequencies. Just as the Kevin Voigt model, the Zener model is unable to capture the frequency dependence of the phase angle. Koeller [14] used Generalized Maxwell Model, which would refer to a spring in parallel with respectively Maxwell cells, to describe the frequency dependence of dynamic stiffness of the viscoelastic components. However the dynamic characteristics of viscoelastic components are often very complex in nature, due to the fact that the response is dependent not only on frequency but also several variables, such as amplitude, preload, and 
temperature which can be certainly critical in capturing the mechanical proprieties and non linear dynamical behaviour appears. Consequently, various methods treating viscoelastic non linear dynamics have been developed. Volterra model, see Schetzen [26], is used in the work of Saad [25] to predict amplitude dependency observed experimentally and to linearize a viscohyperelastic model to take preload effects into account. The non linear dynamic behaviour of preloaded multilayer plates incorporating visco-hyperelastic material confined between stiff layers and worked as a damping layer is investigated by Gacem [11]. Monsia [17] proposed a non linear generalized Maxwell model which consisting of a non linear spring connected in series with a non linear dashpot obeying a power law with constant material parameters, for representing the time-dependent properties of a variety of viscoelastic materials. Monsia [18] developed a non linear mathematical model with constant material coefficients applicable for characterizing the time-dependent deformation behaviour of a variety of materials under a constant loading.

In this context, this paper introduced a new approach for non linear Generalized Maxwell Model in order to describe the dynamic behaviour of viscoelastic components. DMA tests have been conducted in order to identify parameters of the proposed NLGMM which shows a good accuracy when a comparison between experiments and simulations is performed.

The planning of the present paper is as follows: in section 2, a description of the experimental procedure to characterize the viscoelastic component is presented. The proposed NLGMM and the identification techniques of its linear and non linear parameters are detailed in section 3. Comparison between identified and measured values is also performed. In section 4, the validity of the NLGMM is investigated and discussed.

\section{EXPERIMENTAL CHARACTERIZATION}

When a material is subjected to a sinusoidal cyclic displacement of angular frequency $\omega$ :

$$
x(t)=x_{00} \sin (\omega t)
$$

The force response is sinusoidal at the same frequency but with a dephasing angle $\varphi$, called loss angle:

$$
F(t)=F_{00} \sin (\omega t+\varphi)
$$

Generally, this assumption, called the first harmonic, is not sufficient. Typically, the force response contains higher order harmonics, and the real response is expressed as follows, see Long [16]:

$$
F(t)=\sum_{k} F_{k} \sin \left(k \omega t+\varphi_{k}\right)
$$

In the case of the assumption of the first harmonic, the complex stiffness $K^{*}(\omega)$ relates the Fourier transform of the imposed displacement $\hat{x}(\omega)$ to the corresponding force $\hat{F}(\omega)$ is defined as follows:

$$
\hat{F}(\omega)=K^{*}(\omega) \hat{x}(\omega)
$$

with the Fourier transform :

$$
\begin{gathered}
\hat{x}(\omega)=\int_{-\infty}^{+\infty} x(t) \exp (-j \omega t) d t \\
\hat{F}(\omega)=\int_{-\infty}^{+\infty} F(t) \exp (-j \omega t) d t
\end{gathered}
$$

The dynamic stiffness is defined as:

$$
\begin{aligned}
K^{*}(\omega) & =\frac{\hat{F}(\omega)}{\hat{x}(\omega)}=\frac{F_{00}}{x_{00}} \cdot \exp (j \varphi) \\
& =K^{\prime}(\omega)+j K^{\prime \prime}(\omega)=K^{\prime}(\omega)[1+j \tan \varphi]
\end{aligned}
$$

\subsection{EXPERIMENT}

The DMA tester is a bench test performed to characterize the behaviour of dynamic compression of a natural rubber sample as shown in Fig 1.

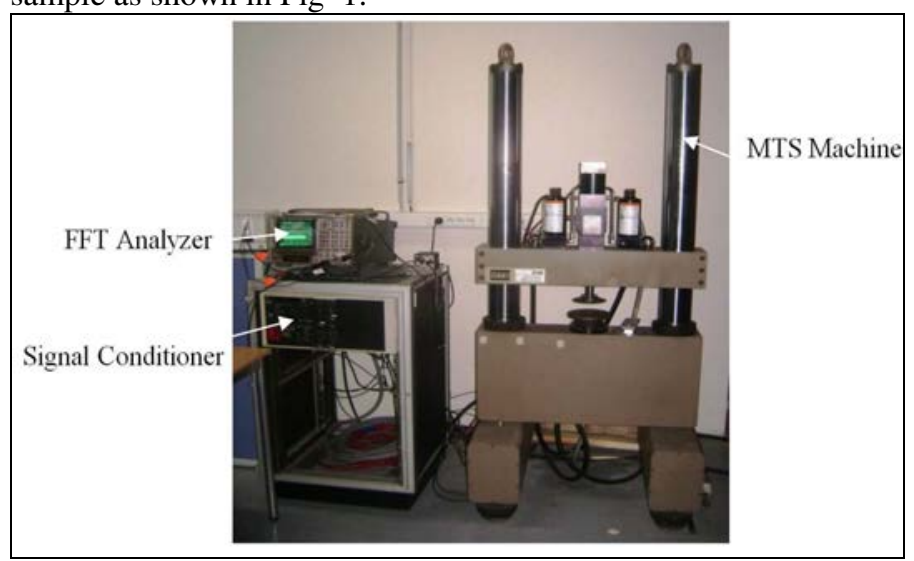

Figure 1. THE MTS BENCH TEST.

The cylindrical elastomeric sample is subjected to uniaxial compression tests. The mechanical solicitation is performed using a hydraulic cylinder with a LVDT (Linear Variable Differential Transformer) displacement sensor. The system is also equipped with a force sensor built into the base of the assembly apparatus. The force and displacement signals after analog conditioning are returned on a spectrum analyzer for digital processing. The entire system is controlled by a computer equipped with an interface GPIB (General Purpose Interface Bus) card connected to the FFT (Fast Fourier Transform) analyzer which manages and controls the sweeping frequencies of the signal by incrementing the excitation frequency for each acquisition.

To determine dynamic stiffness $K^{*}$ of a material, the sample is placed between two rigid surfaces. Surfaces are flat and parallel as presented in Fig 2. 


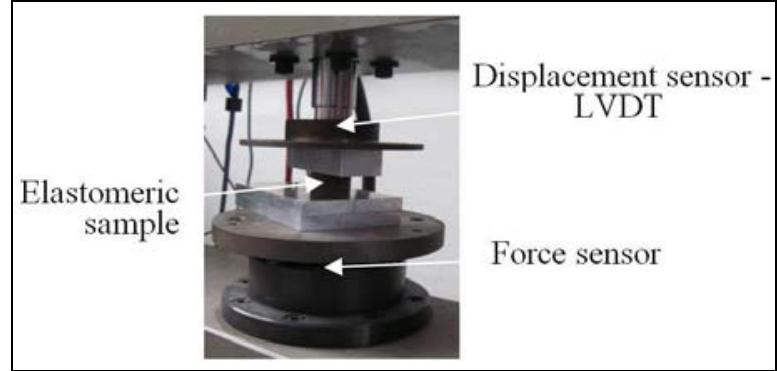

Figure 2. UNIAXIAL COMPRESSION TEST.

During these unidirectional tests, devices measure the vertical force imposed and $\Delta H$ the vertical displacement of upper surface of the rubber sample. For a cylindrical rubber sample of height $H_{0}=13 \mathrm{~mm}$ and diameter $D_{0}=28 \mathrm{~mm}$, the surface on which the force acts is $S_{0}=\pi \times\left(D_{0} / 2\right)^{2}$. The dynamic stiffness is given by:

$$
K^{*}=\frac{F}{\Delta H}
$$

Tests are carried out to evaluate the dynamic behaviour of elastomeric sample and are performed by applying a mechanical sinusoidal solicitation. Elastomeric materials present a different behaviour according to the amplitude of sinusoidal displacement and conforming to the imposed static preload, see $[13,19,25,27]$.

These tests are carried out for different static preloads $\mathrm{P}=$ $[300,500,700,1000,1500,2000,2500] \mathrm{N}$ and different amplitudes $A=[10,25,60,100,150,250,600] \mu \mathrm{m}$. Measuring devices allows calculating the dynamic stiffness for frequencies ranging from 4 to $130 \mathrm{~Hz}$. For each test, the component is submitted to many excitation cycles. Measures were taken when cycles were stable and the stability of the cycle is expected to avoid taking into account the disturbance due to transitory states.

Experiments were performed at room temperature $\mathrm{T}=20^{\circ} \mathrm{C}$.

\subsection{RESULTS}

The dynamic stiffness magnitude and phase angle are used here to characterize the dynamic properties. Harmonic dynamic tests have been performed to analyze the amplitude, preload and frequency dependence of the dynamic stiffness and phase angle. An overview of the results is shown in Fig 3 and Fig 4.

Each plan presents the preload in Fig 3 and the amplitude in Fig 4. Fig 3 illustrates that the dynamic stiffness magnitude shows considerable dependence to amplitude. The magnitude declines when amplitude increases towards an asymptotic value for large amplitudes. The phase angle reaches a maximum when the magnitude stiffness decreases to its minimum at maximal amplitude. This phenomenon identified since 1965 is called Payne effect, see Gacem [11]. Fig 4 shows that with raising preloads, the dynamic stiffness magnitude raises and no effect is shown for the phase angle. Imposed preloads and amplitude of displacement have opposed effects. In fact, the rubber shows a softening behaviour with increasing amplitudes as well as a hardening behaviour with increasing preloads. Dynamic stiffness magnitude and angle phase raises with increasing frequency.

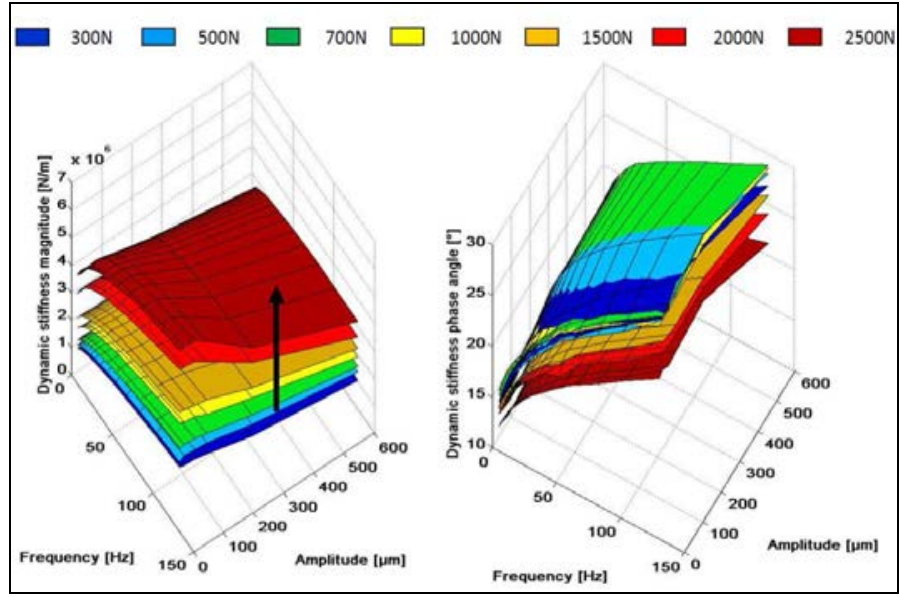

Figure 3. DYNAMIC STIFFNESS MAGNITUDE AND

PHASE ANGLE AS FUNCTION OF AMPLITUDE AND FREQUENCY FOR EACH PRELOAD.

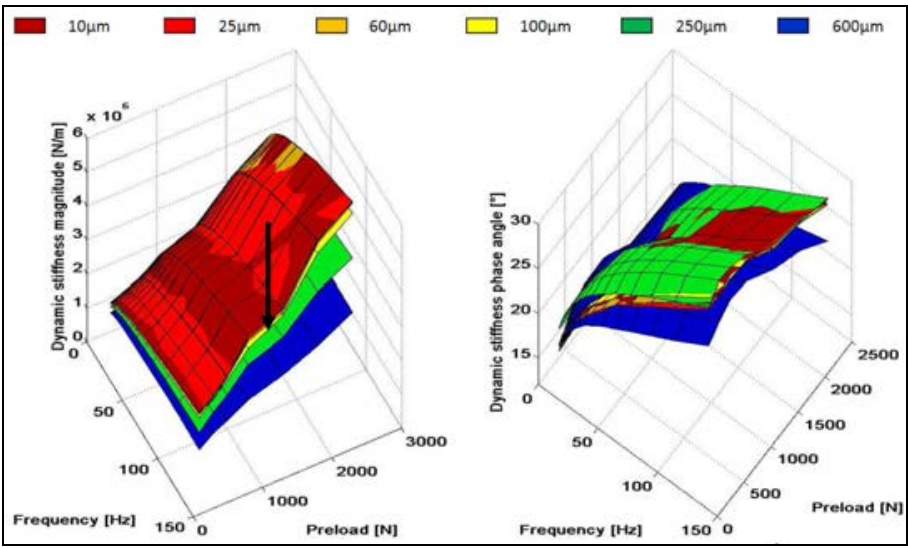

Figure 4. DYNAMIC STIFFNESS MAGNITUDE AND

PHASE ANGLE AS FUNCTION OF PRELOAD AND FREQUENCY FOR EACH AMPLITUDE.

\section{NON-LINEAR GENERALIZED MAXWELL MODEL AND PARAMETRIC IDENTIFICATION TECHNIQUES}

\subsection{NON-LINEAR GENERALIZED MAXWELL MODEL}

Generalized Maxwell Model (GMM) allows an accurate description of the dynamic behaviour of a viscoelastic material. Generalized Maxwell Model is classically composed of Maxwell cells in parallel. A Maxwell cell is represented by a spring and dash-pot connected in series. With such definition 
this model is not able to display reversible creep, see [3]. As this paper deals only with viscoelastic solids, NLGMM would refer to a spring in parallel with respectively Maxwell cells, see [14]. Thus, the GMM defined here is the same as that used by [7] and the same as the Maxwell representation given by Caputo and Mainardi [3], without the first dashpot.

To model the non-linear dynamic behaviour of the rubber, the chosen NLGMM is composed of a non-linear spring and $\mathrm{N}$ linear Maxwell cells as represented in Fig 5.

To deform this rheological model, it is necessary to impose a displacement $x(t)$, the response is the sum of the non-linear spring force added to each cell reaction, noted $F(t)$ :

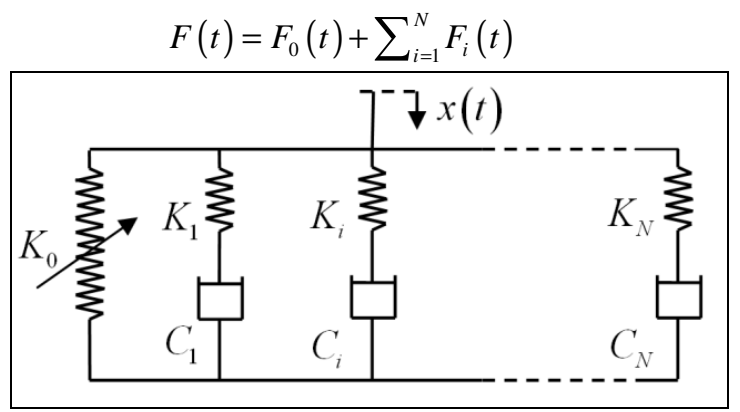

Figure 5. THE PROPOSED NLGMM.

The rheological formulation of the dynamic stiffness of NLGMM is

$$
Z(\omega)=K_{0}+\sum_{i=1}^{N} \frac{j \omega K_{i} C_{i}}{K_{i}+j \omega C_{i}}
$$

$K_{0}$ is the stiffness taken at $\omega=0$, ie. $t=+\infty, K_{i}$ is the stiffness of the $i^{\text {th }}$ spring and $C_{i}$ is the damping of the $\mathrm{i}^{\text {th }}$ dashpot.

Reducing Eqn. (10) to the same denominator and grouping monomials gives the dynamic stiffness of NLGMM expressed as the ratio of two polynomials of the same degree $N$ (number of Maxwell cells). This formulation of transfer function is also used in automation, namely, Oustaloup [21] provided a model using poles and zeros formulation (PZF).

$$
Z(\omega)=K_{0} \prod_{i=1}^{N}\left(\frac{1+\left(j \omega / \omega_{z, i}\right)}{1+\left(j \omega / \omega_{p, i}\right)}\right)
$$

$\omega_{z, i}$ and $\omega_{p, i}$ are respectively the zero and the pole of the $i^{t h}$ Pole-Zero couple, $i \in[1 . . N]$. This operator called by Oustaloup [21] "CRONE regulator" facilitates considerably the treatment and the parametric identification of the polynomial ratio by expressing it in the form of products.

\subsection{PARAMETRIC IDENTIFICATION TECHNIQUES}

The prediction of dynamic behaviour is directly linked to parameters of rheological model. In this section the parametric identification method has been built to provide accuracy and can be automatically executed for a broad-based measurement tests. Renaud [23] demonstrated relations in Eqn. (12) which allow computing NLGMM parameters, given by Eqn. (10), from the parameters of PZF (Poles-Zeros Formulation).

$$
\left\{\begin{array}{l}
K_{i}=\overline{K_{0}} \prod_{h=1}^{N}\left(\frac{\omega_{p, h}}{\omega_{z, h}}\right)\left(\frac{\omega_{p, i}-\omega_{z, h}}{\omega_{p, i}+\omega_{p, h}\left(\delta_{i h}-1\right)}\right) \\
C_{i}=\frac{K_{i}}{\omega_{p, i}}
\end{array}\right.
$$

The dampers $C_{i}$ and the stiffnesses $K_{i}$ of the Maxwell model are commons for all cells. $K_{i}$ and $C_{i}$ coefficients are identified through Eqn. (12.a) and (12.b), after computing the poles and zeros.

Considering $N=5$ Maxwell cells, Fig 6 shows a diagram that summarizes all the steps performed for identification of 13 parameters (10 for the linear viscoelastic model and 3 for the non linear elasticity). The following identification method aims to reduce the number of parameters to 4 independent and identifiable parameters.

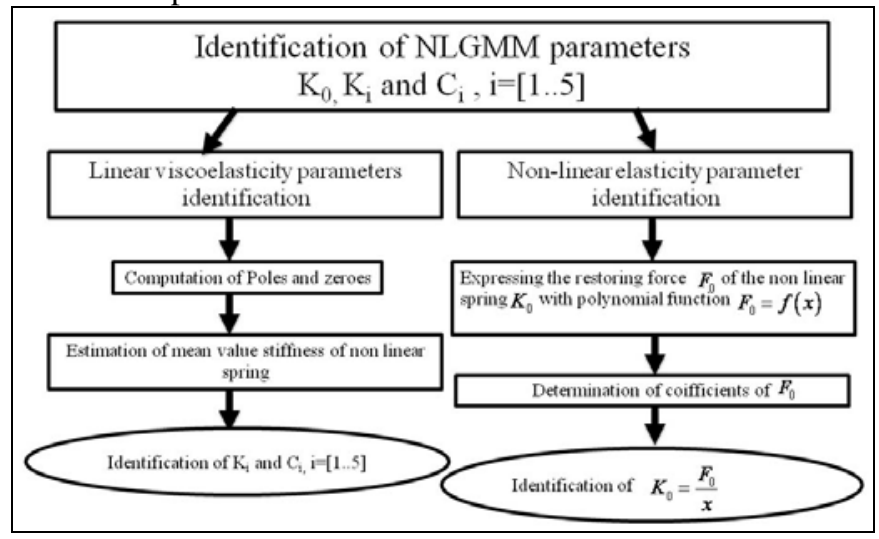

Figure 6. SUMMARY OF NLGMM PARAMETERS IDENTIFICATION STEPS.

\subsection{1. $\mathrm{Ki}$ and $\mathrm{Ci}$ identification}

The method used to determine the poles and zeros is analogous to the one proposed by Oustaloup [21]. The main idea of this approach leads to consider the angle phase equal to $\pi / 2$ between zero and pole of the same order and null elsewhere. The resulting phase angle in the studied frequency range is then estimated as the average of phase angle calculated between the first zero and the last pole, see Dion [8,9].

To obtain a constant phase between two consecutive zeros, the ratio between two consecutive zeros is constant and equal to the ratio between two consecutive poles. Two constants $\lambda$ and $\theta$ are then defined:

$$
\ln (\lambda)=\phi \frac{\ln \left(f_{2}\right)-\ln \left(f_{1}\right)}{\phi+\frac{\pi}{2}(N-1)}
$$




$$
\ln (\theta)=\left(\frac{\pi}{2}-\phi\right) \frac{\ln \left(f_{2}\right)-\ln \left(f_{1}\right)}{\phi+\frac{\pi}{2}(N-1)}
$$

$f_{1}$ and $f_{2}$ are respectively the upper and lower bounds of the frequency domain on which $\phi$ the mean phase angle for all tests is identified and $\ln (y)$ is the natural logarithm of $y$. The first zero coincides with $f_{1}$ and the last pole with $f_{2}$. This approach is illustrated in Fig 7.

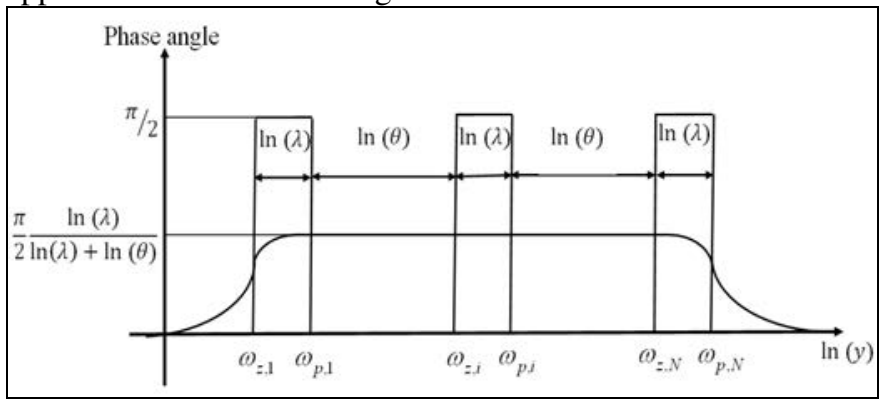

Figure 7. APPROACH OF CONSTANT PHASE ANGLE.

The identification of a viscoelastic behaviour can be performed using a ratio of two polynomial functions defined by zeros and poles.

Zeros are defined from the first zero, so that

$$
\omega_{z, i+1}=\omega_{z, i} \lambda \theta
$$

Poles are calculated from the last pole, so that

$$
\omega_{p, i}=\frac{\omega_{p, i+1}}{\lambda \theta}
$$

The relation between zeros and poles is given by

$$
\omega_{p, i}=\lambda \omega_{z, i}
$$

The mean value of estimated stiffness of non linear spring is then computed

$$
\bar{K}_{0}=\int_{\omega}\left(\prod_{i=1}^{N}\left(\frac{1+\left(j \omega / \omega_{z, i}\right)}{1+\left(j \omega / \omega_{p, i}\right)}\right) / K_{m}^{*}(\omega)\right) d \omega
$$

with $K_{m}^{*}(\omega)$ are measures of dynamic stiffness.

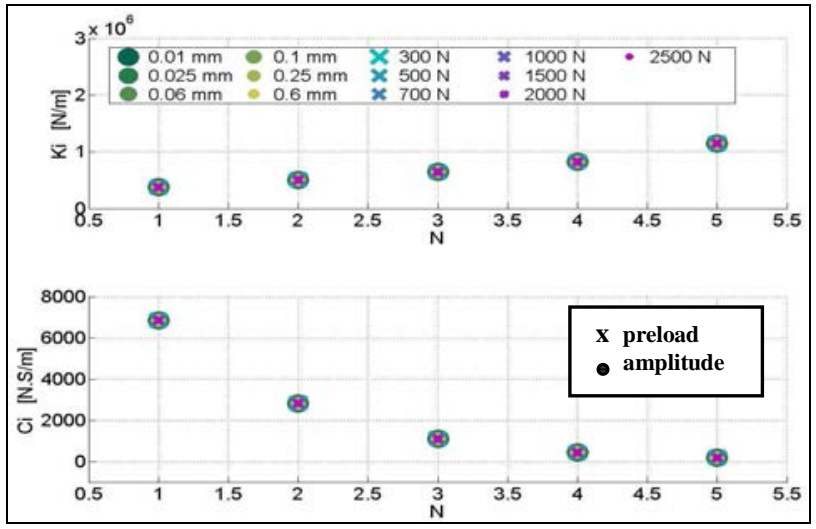

Figure 8. IDENTIFIED $K_{\mathrm{i}}$ AND $C_{\mathrm{i}}$.
Fig 8 shows that the dampers $C_{i}$ and the stiffnesses $K_{i}$ of the NLGMM are commons for all cells with all tested amplitudes and static preloads.

\subsection{2. $\mathrm{KO}$ identification}

The displacement is proposed to be expressed as:

$$
x(t)=x_{0}+x_{d} \sin (\omega t)
$$

$x_{0}$ is the displacement under static preload.

$x_{d}$ is the amplitude of displacement.

Non linear spring stiffness depends not only on static solicitations but also on dynamic ones as it is represented in Fig 9. We proposed to model the problem with only one curve $F_{0}=f(x)$. Indeed, dynamics of rubber are integrated in the non linear function $F_{0}$. Fig 10 illustrates the researched function between force and displacement.

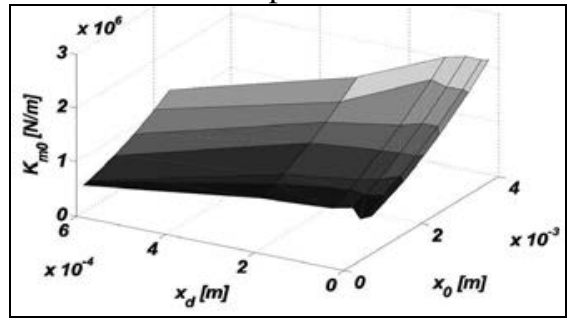

Figure 9. NON LINEAR SPRING STIFFNESS DEPENDING OF STATIC AND DYNAMIC EXCITATION.

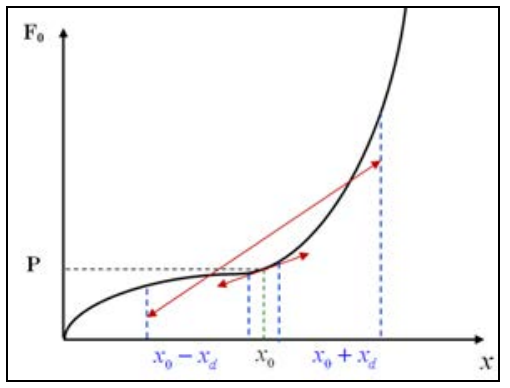

Figure 10. DETERMINATION OF $K_{0}$ : SKETCH OF THE NON LINEAR RELATION BETWEEN FORCE AND DISPLACEMENTS.

The identification of $K_{0}$ is based on the assumption of a bijective function between the force and the displacement (Fig 10) able to describe the whole surface represented in Fig 9.For small dynamic displacements, $K_{0}$ can be considered as the local tangent at $x_{0}$. However, for larger amplitudes $x_{d}, K_{0}$ is estimated by calculating the average slope in the range of dynamic excitation $\left[x_{0}-x_{d}, x_{0}+x_{d}\right]$.

The resorting force $F_{0}$ of the non linear spring $K_{0}$ is proposed to be expressed as

$$
F_{0}(t)=\alpha x^{3}(t)+\beta x^{2}(t)+\gamma x(t)
$$

with $\alpha, \beta$ and $\gamma$ are real constants. 
The expression of $F_{0}$ takes into account a quadratic term. This choice is due to analysis of the dynamic behaviour of the rubber sample which shows softening behaviour observed with increasing amplitudes of the excitation, and the hardening for increasing preloads.

Substituting Eqn. (19) in Eqn. (20), the following expression is obtained:

$$
\begin{aligned}
F_{0}(t) & =x_{d}^{3}\left(\alpha\left(\frac{3}{4} \sin (\omega t)-\frac{1}{4} \sin (3 \omega t)\right)\right) \\
& +x_{d}^{2}\left(3 \alpha x_{0}+\beta\right)\left(\frac{1}{2}-\frac{\cos (2 \omega t)}{2}\right) \\
& +x_{d}\left(3 \alpha x_{0}^{2}+2 \beta x_{0}+\gamma\right) \sin (\omega t)+3 x_{0}^{3}+\beta x_{0}^{2}+\gamma x_{0}
\end{aligned}
$$

Measurement process of the stiffness is only based on the excitation frequency $\omega$. Thus, the measured force is:

$$
F_{0}(t)=\sin (\omega t)\left(\frac{3}{4} \alpha x_{d}^{3}+\left(3 \alpha x_{0}^{2}+2 \beta x_{0}+\gamma\right) x_{d}\right)
$$

and $K_{0}$ has the following form:

$$
K_{0}=\frac{F_{0}(t)}{x_{d} \sin (\omega t)}=\frac{3}{4} \alpha x_{d}^{2}+3 \alpha x_{0}^{2}+2 \beta x_{0}+\gamma
$$

Having $x_{0}$ and $x_{d}$ measured $\alpha, \beta$ and $\gamma$ are determined with minimization in the least square sense by solving the following system of $\mathrm{Ne}$ equations

$$
\left[\begin{array}{c}
K_{\mathrm{m} 01} \\
\vdots \\
K_{\mathrm{m} 0 i} \\
\vdots \\
K_{\mathrm{m} 0 N}
\end{array}\right]=\left[\begin{array}{ccc}
\frac{3}{4} x_{d 1}^{2}+3 x_{01}^{2} & 2 x_{01} & 1 \\
\vdots & \vdots & \vdots \\
\frac{3}{4} x_{d i}^{2}+3 x_{0 i}^{2} & 2 x_{0 i} & 1 \\
\vdots & \vdots & \vdots \\
\frac{3}{4} x_{d N}^{2}+3 x_{0 N}^{2} & 2 x_{0 N} & 1
\end{array}\right]\left[\begin{array}{c}
\alpha \\
\beta \\
\gamma
\end{array}\right]
$$

With $i \in[1 . . N e], N$ is the number of tests. $K_{m 0 i}$ is measured non linear spring stiffness of the $i^{\text {th }}$ test. $K_{0}$ is well identified for $\alpha=2.6410^{10}, \beta=7.1010^{7}$ and $\gamma=4.6310^{5}$.

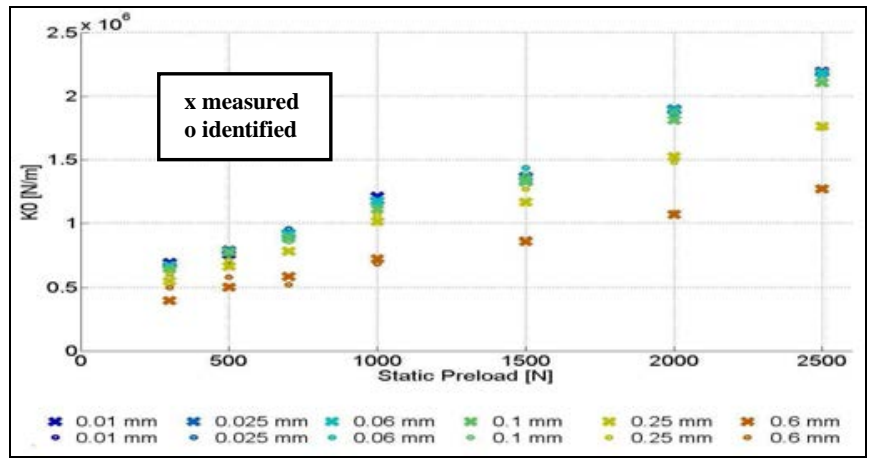

Figure 11. $K_{0}$ AS FUNCTION OF PRELOADIDENTIFICATION FOR DIFFERENT AMPLITUDES.
Fig 11 and 13 show a good agreement between measured and simulated values of $K_{0}$ for different preloads and amplitudes. Relative errors between measured and simulated values of $K_{0}$ are illustrated in Fig 12 and 14. The correlation coefficient between measures and simulations is 0.95 .

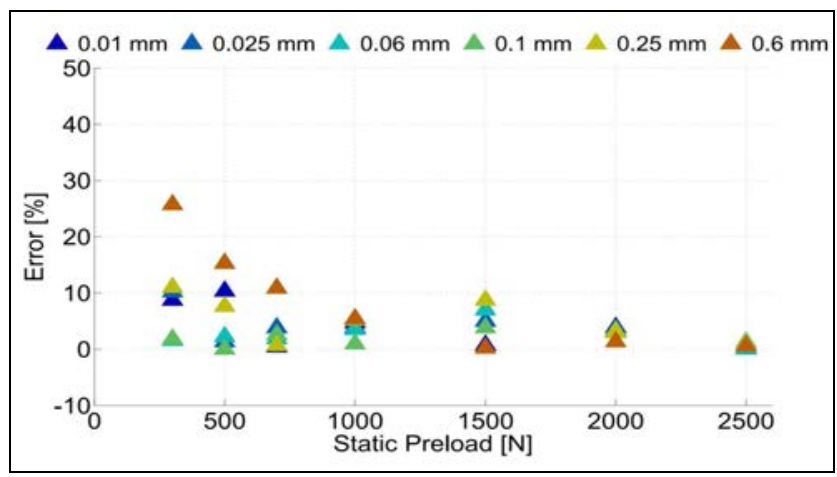

Figure 12. ERROR BETWEEN MEASURED AND SIMULATED $K_{0}$ AS FUNCTION OF STATIC PRELOAD FOR DIFFERENT AMPLITUDES.

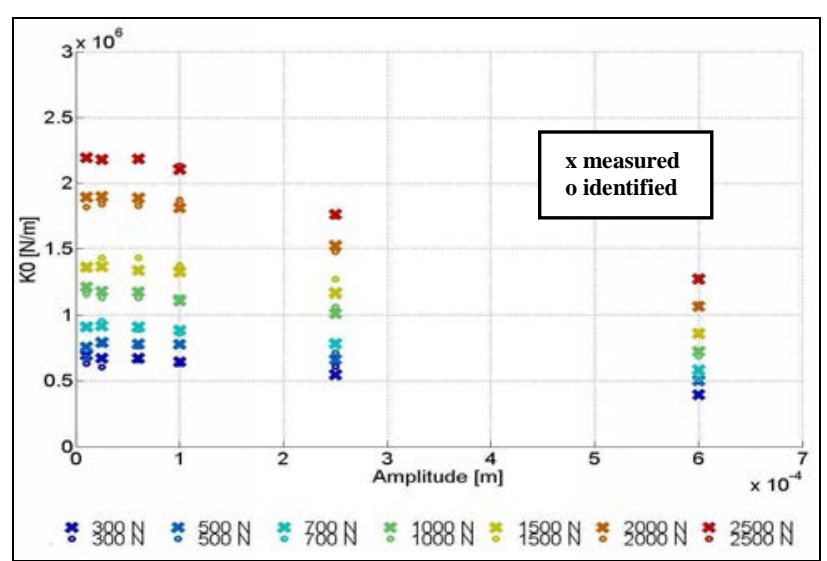

Figure 13. $K_{0}$ AS FUNCTION OF AMPLITUDEIDENTIFICATION FOR DIFFERENT PRELOADS.

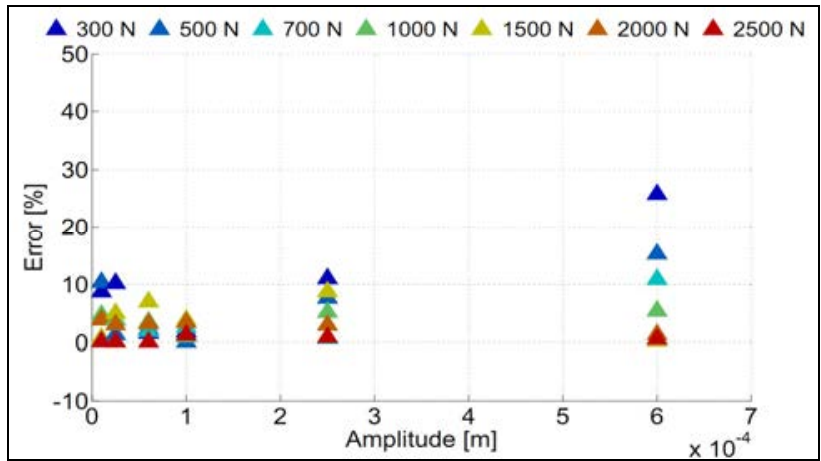

Figure 14. ERROR BETWEEN MEASURED AND SIMULATED $K_{0}$ AS FUNCTION OF AMPLITUDE FOR DIFFERENT PRELOADS. 
According to the curve of $F_{0}$ represented in Fig 15, table 1 presents measured and identified values of $F_{0}$ for static deflection $x_{0}$. Measured values are obtained after each experiment in recording static deflection and force. These measures are close to the real static stiffness. Identified values are the forces computed with the non linear model (Eqn. 20). These values are obtained from dynamic experiments. They are greater than real static one. This difference is well known in parametric identification of viscoelastic model as GMM. This difference is mainly due to the tendency of the solid material to move slowly and to deform under the influence of stresses: creep phenomenon.

Table 1. IDENTIFIED AND MEASURED $x_{0}$ FOR EACH PRELOADS.

\begin{tabular}{llllllll}
\hline Static preload [N] & 300 & 500 & 700 & 1000 & 1500 & 2000 & 2500 \\
\hline Measured $x_{0}[\mathrm{~mm}]$ & 0.69 & 1.08 & 1.35 & 1.92 & 2.41 & 3.04 & 3.49 \\
Calculated $x_{0}[\mathrm{~mm}]$ & 0.63 & 0.92 & 1.20 & 1.5 & 2.11 & 2.51 & 2.86 \\
Error [\%] & 8.69 & 14.81 & 11.11 & 21.87 & 12,44 & 17.43 & 18.05 \\
\hline
\end{tabular}

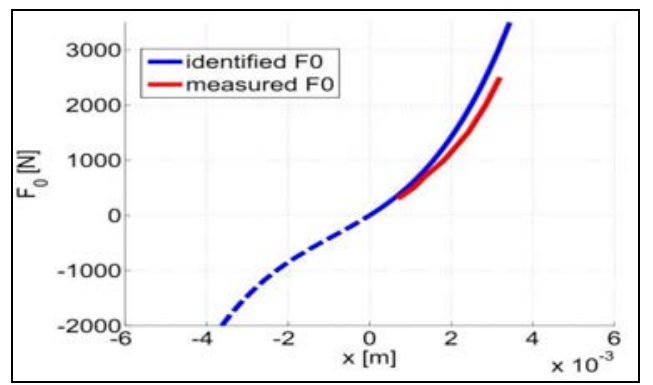

Figure 15. IDENTIFIED AND MEASURED RESORTING FORCE $F_{0}$ OF THE NON-LINEAR SPRING.

\section{VALIDATION RESULTS}

By frequency sweeping, a transfer function between force and displacement can be build on a wide frequency range. Given an experimental transfer function characterizing the dynamic stiffness, the validation of the identified NLGMM can be carried out with some graphical methods. Renaud [24] presented a method based on characteristics of the asymptotes of Pole-Zero formulations which allows identifying NLGMM parameters from both the magnitude and the phase curves with more efficiency than the classical graphical methods thanks to optimization algorithm based on asymptotes.

The dynamic stiffness of NLGMM is well described by its magnitude and phase $Z(\omega)=|Z(\omega)| \exp (j \varphi(\omega))$, magnitude and phase of the associated PZF are defined in Eqn. (25), see [24].

$$
\left\{\begin{array}{l}
|Z(\omega)|=K_{0} \prod_{i=1}^{N}|Z(\omega)|_{i}=K_{0} \prod_{i=1}^{N} \frac{\sqrt{1+\left(\omega / \omega_{z, i}\right)^{2}}}{\sqrt{1+\left(\omega / \omega_{p, i}\right)^{2}}} \\
\varphi(\omega)=\sum_{i=1}^{N} \varphi_{i}(\omega)=\sum_{i=1}^{N}\left(\tan ^{-1}\left(\frac{\omega}{\omega_{z, i}}\right)-\tan ^{-1}\left(\frac{\omega}{\omega_{p, i}}\right)\right)
\end{array}\right.
$$

The NLGMM model is validated in Fig from 16 to 19 . The 3-D plots in Fig 16 and 18: (a) and (b) give an overview of the measured behaviour represented by the colored map and the identified behaviour represented by the transparent map, Fig (c) and (d) show the relative error between measured and identified values. The 2-D plots in Fig 17 and 19 give more detailed information.

Measured and identified values show good agreement. The NLGMM is able to identify perfectly the frequency dependence. The behaviour for low amplitudes is predicted more accurately than the behaviour for high amplitudes. The amplitude $0.6 \mathrm{~mm}$ is the most difficult amplitude to model with respect to both magnitude and angle phase of the dynamic stiffness as it is illustrated in Fig 18 and 19 and the mean relative error for magnitude is $12.31 \%$ and $1.16 \%$ for angle phase for $P=2000 \mathrm{~N}$.

The behaviour for low preloads is predicted more accurately than the behaviour for high preloads. The dynamic stiffness magnitude is underestimated for low frequencies and overestimated for high frequencies for preload $2500 \mathrm{~N}$ as illustrated in Fig 16 and 17 and the mean relative error is for magnitude is $6.66 \%$ and $0.67 \%$ for angle phase for $A=250 \mu \mathrm{m}$.

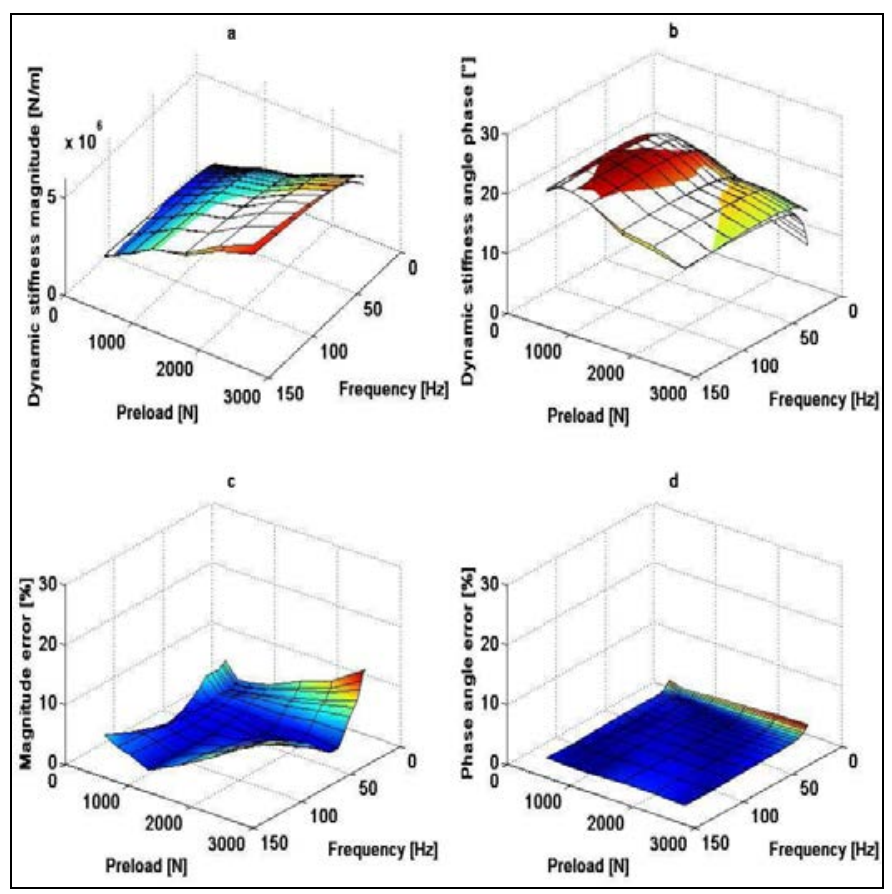

Figure 16. 3-D PLOTS OF DYNAMIC STIFFNESS AS FUNCTION OF FREQUENCY AND PRELOAD FOR A= $250 \mu \mathrm{M}$. (a) MAGNITUDE OF THE DYNAMIC STIFFNESS. (b PHASE ANGLE OF THE DYNAMIC STIFFNESS. (c) RELATIVE ERROR OF THE MAGNITUDE OF THE DYNAMIC STIFFNESS. (d) RELATIVE ERROR OF THE PHASE ANGLE OF THE DYNAMIC STIFFNESS. 


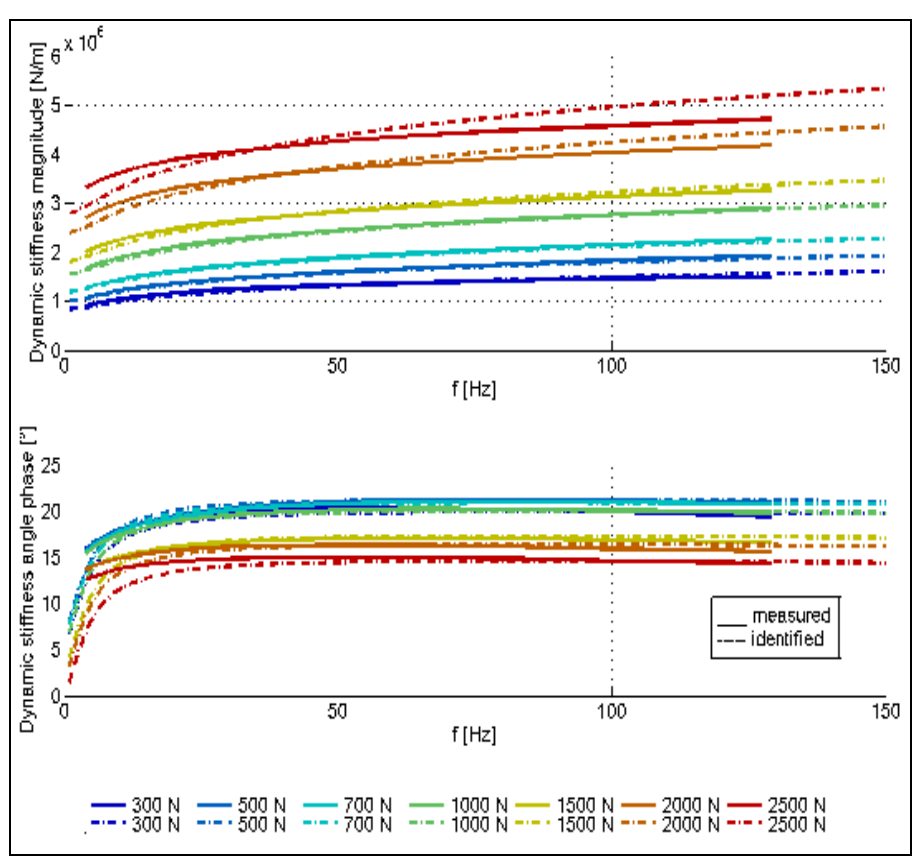

Figure 17. 2-D PLOT OF DYNAMIC STIFFNESS MAGNITUDE AND PHASE ANGLE AS FUNCTION OF FREQUENCY FOR A= 250 $\mu \mathrm{M}$.

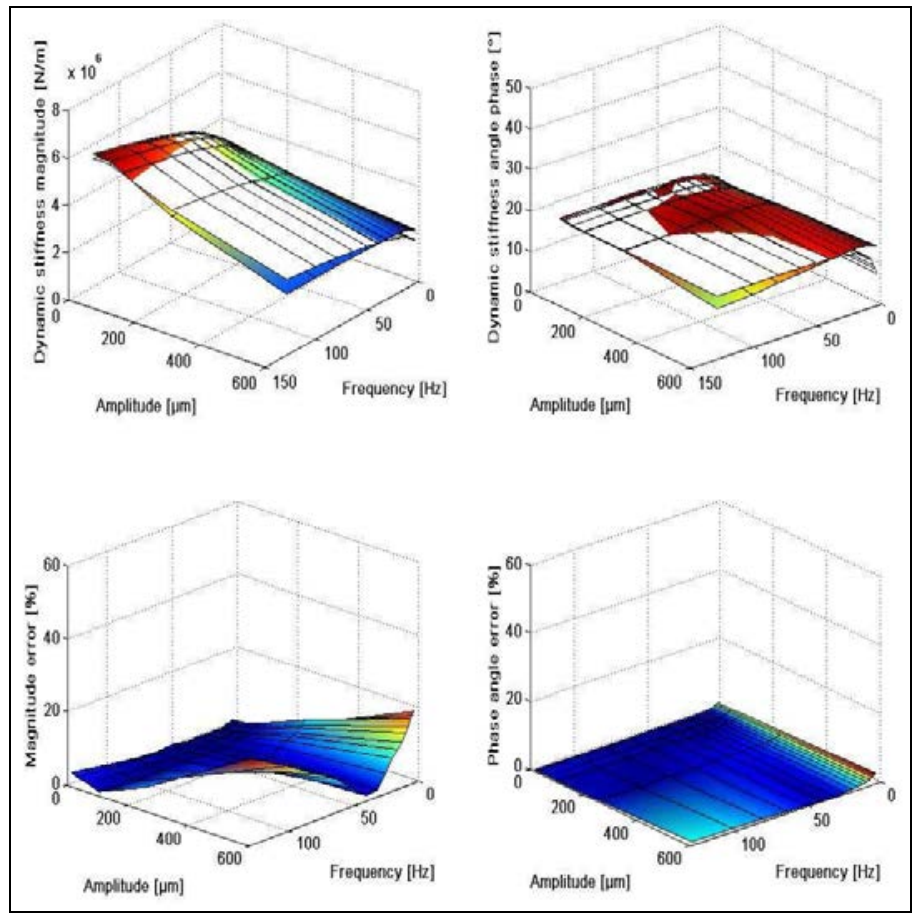

Figure 18. 3-D PLOTS OF DYNAMIC STIFFNESS AS FUNCTION OF FREQUENCY AND AMPLITUDE FOR P= 2000N. (a) MAGNITUDE OF THE DYNAMIC STIFFNESS. (b) PHASE ANGLE OF THE DYNAMIC STIFFNESS. (c) RELATIVE ERROR OF THE MAGNITUDE OF THE
DYNAMIC STIFFNESS. (d) RELATIVE ERROR OF THE PHASE ANGLE OF THE DYNAMIC STIFFNESS.

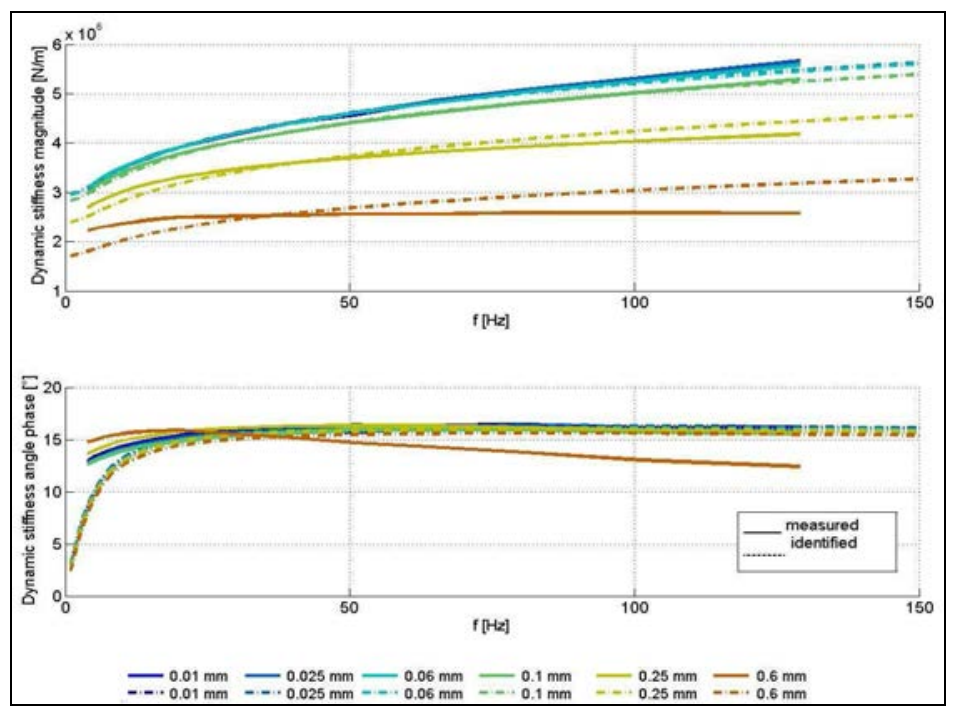

Figure 19. 2-D PLOT OF DYNAMIC STIFFNESS MAGNITUDE AND PHASE ANGLE AS FUNCTION OF FREQUENCY FOR $\mathrm{P}=2000 \mathrm{~N}$.

The NLGMM model describes the behaviour of rubber specimen with satisfying accuracy. The identification method proposed is robust and has been applied for a very large number of tests with several amplitudes and different static preloads. The quality of fitting between simulation and measurements illustrated in the different Fig is similar for most tests.

\section{CONCLUSION}

Viscoelastic components are a key element in designing desired dynamic behaviour of mechanical system; therefore, it is of a great interest to perform studies of the dynamic behaviour of these components in order to refine and develop more advanced models in Multi-body simulations of complete mechanical subsystems.

The proposed NLGMM with only 4 independent parameters $(\phi, \alpha, \beta$ and $\gamma)$ allows an accurate description and good knowledge on the dynamic behaviour of viscoelastic components versus amplitude, preload and wide frequency range. Moreover, it needs only 4 parameters for identifying and it can be used with different kinds of solicitations: harmonic, transient and random signals. The different parameters of the present model can be identified with only one practical test. An accurate method for parametric identification is performed and a good conformity is shown between measurements and simulations.

NLGMM describes faithfully both modulus and phase of complex stiffness characterizing viscoelastic materials and represent very well the softening behaviour with increasing amplitudes as well as a hardening behaviour in increasing preloads. 


\section{REFERENCES}

[1] Balmès, E. and Leclère, J. M., 2009, Viscoelastic vibration toolbox. SDTools, Paris.

[2] Barbosa, F. and Farage, M., 2008, "A finite element model for sandwich viscoelastic beams : Experimental and numerical assessment," Journal of Sound and Vibration, vol. 317, no. 1-2, pp. 91 - 111.

[3] Caputo, M. and Mainardi, F., avril 1971, "Linear models of dissipation in anelastic solids," in La Rivista del Nuovo Cimento (1971-1977) (I. P. Society, ed.), vol. 1, pp. 161-198, Italian Physical Society.

[4] Castello, D., Rochinha, F., Roitman, N. and Magluta, C., 2008, "Constitutive parameter estimation of a viscoelastic model with internal variables," Mechanical Systems and Signal Processing, vol. 22, no. 8 , pp. $1840-1857$.

[5] Chen, T. , may 2000, "Determining a prony series for a viscoelastic material from time varying strain data," tech. rep., NASA.

[6] Chevalier, Y., 2002, "Essais dynamiques sur composites. caractérisation aux basses fréquences.," tech. rep., Techniques de l'ingénieur.

[7] Chevalier, Y. and Vinh, J. T. , 2010, Mechanics of Viscoelastic Materials and Wave Dispersion, vol. 1. ISTE and John Wiley \& Sons.

[8] Dion, J. L., 1995 ,"Modélisation et identification du comportement dynamique de liaisons hydroélastiques", Ph.D. Thesis, ISMCM, Paris.

[9] Dion, J. L., Vialard, S., 1997, "Identification or rubber shock absorber mounts", Mécanique industrielle et matériaux 50 232- 237.

[10] Ferry, J. D. , 1961, "Viscoelastic Properties Of Polymers". John Wiley \& Sons.

[11] Gacem, H. ,2007, "Hyperelastic Behaviour of Elastomers, Non linear Viscoelasticity, Application on Multilayers," Ph.D. thesis, LISMMA.,SUPMECA, Paris.

[12] Gaul, L. , Klein, P. and Kemple, S., 1991, "Damping description involving fractional operators,"Mechanical Systems and Signal Processing, vol. 5, no. 2, pp. 81 88.

[13] Huynh, A., Argoul, P., Point, N. and Dion, J. L., 2002, " Rheological models using fractional derivatives for linear viscoelastic materials: Application to identification of the behaviour of elastomers," 13th Symposium Vibration, Shock \& Noise, Lyon.

[14] Koeller, R. C., 1984, "Applications of fractional calculus to the theory of viscoelasticity," Journal of Applied Mechanics, 51(2) :299-307.

[15] Lakes, R. S., 1999, “Viscoelastic Solids”. CRC Press.
[16] Long, A. H. K., 2005, "Analysis of the Dynamic Behaviour of an Elastomer: Modeling and Identification," Ph.D. thesis, LISMMA, SUPMECA, Paris.

[17] Monsia, M. D., 2011, "A Simplified Non linear Generalized Maxwell Model for Predicting the Time Dependent Behaviour of Viscoelastic Materials", World Journal of Mechanics, 1, 158-167.

[18] Monsia, M. D., January 2012 ,"A Non linear Mechanical Model for Predicting the Dynamics Response of Materials under a Constant Loading", Journal of Materials Science Research, Vol.1(1) p90.

[19] Moreau, A., 2007, "Identification of viscoelastic properties of polymeric materials by field measurements of frequency response of structures," Ph.D. thesis, LMR., INSA, Rouen.

[20] Oberst, H. and Frankenfeld, K. , 1952, "Damping of the bending vibrations of thin laminated metal beams connected through adherent layer," Acustica 2, pp. 181-194.

[21] Oustaloup, A., 1991, La commande CRONE: commande robuste d'ordre non entier, Hermès.

[22] Park, S.W, 2001, “Analytical modeling of viscoelastic dampers for structural and vibration control," Int. J. of Solids and Struct., 38, pp. 8065 - 8092.

[23] Renaud, F., Dion, J. L., Chevallier, G., Tawfiq, I. and Lemaire, R., 2011, "A new identification method of viscoelastic behaviour: Application to the generalized Maxwell model," Mechanical Systems and Signal Processing, vol. 25, no. 3, pp. $991-1010$.

[24] Renaud, F., 2011, “ Étude de l'incidence des comportements dissipatifs dans les instabilités vibratoires des systèmes de freinage," Ph.D. thesis, LISMMA., SUPMECA, Paris.

[25] Saad, P., 2003, "Non linear Behaviour of Rubber Bush, Modeling and Identification," Ph.D. thesis, LTDS., Ecole Centrale de Lyon, Lyon.

[26] Schetzen, M., 1980, "The Volterra and Wiener theories of non linear systems", John Wiley \& Sons, New York.

[27] Soula, M. and Chevalier,Y., 1998, “ The fractional derivative rheological polymers - application to elastic and viscoelastic behaviours linear and non linear elastomers," ESAIM Proceedings: Fractional Differential Systems 5, 193-204.

[28] Vinh, J. T., 1967, "Sur le passage du régime harmonique au régime transitoire viscoelastique," Mémorial de l'artillerie française, vol. 3ème fascicule. 